ISSN 1997-5902

\title{
Le carbone et l'azote dans les différentes fractions granulométriques d'un sol brun eutrophe tropical sous irrigation de Bagré au Burkina Faso : effets de modes d'apports différents de matières organiques et minérales.
}

\author{
Zacharie SEGDA $^{1,2^{*}}$; Louis P. YAMEOGO1 ${ }^{* *}$, Moussa BONZI ${ }^{3+}$ et Michel P. SEDOGO ${ }^{3++}$ \\ ${ }^{1}$ Institut de l'Environnement et de Recherches Agricoles (INERA), Station de Recherches de Farako-bâ, 01 BP 910 Bobo- \\ Dioulasso 01, Burkina Faso, ${ }^{2}$ Centre Agricole Polyvalent de Matourkou, 01 BP 130 Bobo Dioulasso 01, Burkina Faso. \\ 3Institut de l'Environnement et de Recherches Agricoles (INERA), Station de recherches de Kamboinsé, 04 BP 8645 \\ Ouagadougou 04, Burkina Faso. \\ *Auteur correspondant : E-mail : **ylouis5@yahoo.fr ; cel : +226 71911450 \\ Autres auteurs : E-mail : "zacharie.segda@yahoo.fr ; ' ${ }^{*}$ mous bonz@yahoo.fr; ${ }^{++}$michel.sedogo@yahoo.fr;
}

Original submitted in on 21st January 2014. Published online at www.m.elewa.org on 30thJune 2014. http://dx.doi.org/10.4314/jab.v78i1.6

\section{RESUME}

Objectif : Une expérimentation a été conduite pour étudier l'influence des systèmes de culture (modes d'apports différents de matières organiques et minérales) sur l'évolution qualitative du carbone et de l'azote sur un sol brun eutrophe tropical en conditions de riziculture irriguée à Bagré au Burkina Faso.

Méthodologie et résultats : La méthode de fractionnement physique de la matière organique a été utilisée et a permis de suivre l'évolution du carbone organique et de l'azote total du sol. Les résultats montrent que la mise en culture conduit à une diminution du stock de carbone organique et de l'azote total dans les horizons supérieurs $(0-20 \mathrm{~cm})$ pendant qu'une accumulation est observée dans les horizons inférieurs $(20-50 \mathrm{~cm})$. Dans les horizons inférieurs, l'importance de cette accumulation dépend du niveau de restitution en fumure organique.

Conclusion et application : Contrairement aux résultats enregistrés dans les systèmes pluviaux, la mise en culture conduirait à une accumulation du carbone organique total et de l'azote total dans les sols sous irrigation par rapport à la "jachère". L'exportation même totale des résidus culturaux des rizières n'influencerait pas cette accumulation qui est sous la dépendance de plusieurs facteurs. Ce résultat est d'un grand intérêt dans la gestion de la fumure organique et minérale d'autant plus que l'accumulation du carbone organique suppose en partie une faible minéralisation de la matière organique du sol. Ainsi, pour des cultures irriguées comme le riz beaucoup plus exigeantes en élément minéraux surtout l'azote, l'apport de fertilisants minéraux en combinaison avec la fumure organique bien décomposée pourrait assurer aux plantes une bonne alimentation minérale. Aussi, malgré la production de méthane reconnue pour la riziculture irriguée, elle pourrait tout de même contribuer dans la séquestration du carbone atmosphérique.

Mots clés : carbone, azote, séquestration, sol irrigué 


\section{ABSTRACT}

Objective: A study was carried out to evaluate the influence of cropping systems (modes of different contributions of organic and mineral fertilizers) on the qualitative evolution of soil organic carbon and nitrogen of a tropical eutrophic brown soil under irrigation of Bagré, Burkina Faso.

Methodology and results: The granulometric soil organic matter fractionation method was used and allowed to follow the qualitative evolution of soil organic carbon and total nitrogen. The results show that rice cultivation leads to reduction of soil organic carbon and total nitrogen in the upper horizons $(0-20 \mathrm{~cm})$ while an accumulation is observed in lower horizons $(20-50 \mathrm{~cm})$. In the lower horizons, the importance of accumulation depends of organic manure restitution level.

Conclusion and application: Contrary to results obtained under upland cropping systems, irrigated rice cultivation leads to an accumulation of soil organic carbon and nitrogen compared to "fallow". Even complete crop residues removing in irrigated soil may result in organic carbon accumulation. This result has a great interest in organic and fertilizer management all the more reason that soil organic carbon accumulation result of lower soil organic matter mineralization. Thereby, for irrigated crops like rice which is exigent to nutrients mostly nitrogen, the combination of mineral fertilizers with organic manure well decomposed could allow a better nutrient nutrition. Furthermore, despite the production of methane, irrigated rice system could all the same contribute to atmospheric carbon sequestration.

Key words: carbon, nitrogen, accumulation, irrigated soil

\section{INTRODUCTION}

Les propriétés intrinsèques du sol contribuent largement à la minéralisation du carbone et de l'azote où le $\mathrm{pH}$ joue un rôle important (Wang et al., 2001). Les systèmes d'utilisation des terres ont un grand impact sur le flux de $\mathrm{CO}_{2}$ à partir de la surface du sol (Creamer et al., 2013). La minéralisation du carbone organique du sol avec comme résultante la production de dioxyde de carbone ont un important effet sur le cycle du carbone et le fonctionnement de l'écosystème terrestre (Jenkinson et al., 1991; Valentini et al., 2000; IPCC, 2008). Le carbone organique du sol est un indicateur de la fertilité et de la qualité des terres arables (Abro et al., 2011). II a une première importance dans le cycle des nutriments, l'amélioration des propriétés physiques, chimiques et biologiques des sols, de la productivité des cultures et dans la réduction des gaz à effet de serre (Bhattacharyya, 2009). Le carbone du sol et l'azote sont ainsi utilisés comme indicateurs dans l'évaluation de la qualité des sols et la gestion durable des terres. En effet, le rapport $\mathrm{C} / \mathrm{N}$ est un indicateur de la fertilité des sols et l'évaluation de l'équilibre entre le carbone et l'azote (Zhang et al, 2011; Ge et al. 2013). Quand bien même que le carbone et l'azote à eux seuls ne reflètent pas le niveau de la fertilité des sols, ils peuvent cependant expliquer l'évolution de l'écosystème régional. Plusieurs auteurs ont montré que les teneurs en carbone organique et azote du sol ne sont pas seulement affecté par le climat, mais aussi le système d'utilisation des terres (Sui et al, 2005; Li et al, 2011; Ge et al. 2013).

La réponse du riz aux éléments nutritifs apportés dépend de la fertilité intrinsèque du sol, laquelle est liée à la teneur du sol en matière organique (Segda, 2006). Cependant, la matière organique n'est pas uniformément répartie dans le sol. Elle se retrouve non seulement dans les différents horizons du sol mais aussi pour un même horizon dans les différentes classes granulométriques du sol avec des propriétés physiques, chimiques et biologiques différentes (Feller et al., 1991a et b; Powers \& Schlesinger, 2002). La teneur du sol en carbone dépend des principaux facteurs à long terme de la formation du sol, mais elle peut être fortement modifiée, dégradée ou améliorée par les changements d'utilisation du sol et la gestion du sol (FAO, 2002).Les systèmes de culture affectent différemment le niveau organique des sols caractérisé par les teneurs en carbone organique et azote totaux. En outre, le mode de gestion de la fertilité des sols influence fortement le sens d'évolution du statut organique dans les différentes fractions granulométriques. Des chercheurs de plus en plus nombreux utilisent des méthodes physiques, 
préalablement à toute extraction chimique, pour l'étude de la matière organique (Feller, 1979 ; Elliott et Cambardella, 1991 ; Christensen, 1992 ; Sedogo, 1993 ; Feller and Beare, 1997 ; Sombrero and Benito, 2010 ; Segda et al., 2014). Plusieurs des techniques suivantes sont en général retenues dans un seul protocole: densimétrie dans liqueurs denses organiques ou minérales, flottation dans l'eau, ultrasonication, tamisages, sédimentation, décantation, élutriation, centrifugation. Les objectifs visés peuvent être très divers, à savoir (Feller, 1979): (i) l'estimation de la biomasse des racines ou des fractions organiques dites «légères» (résidus végétaux plus ou moins humifiés), (ii) l'étude de la répartition du carbone et de l'azote dans différentes fractions organiques ou dans les complexes organominéraux (macro et micro-agrégats), (iii) la caractérisation biologique de certaines fractions organiques ou l'étude de l'activité biologique des sols, (iv) la caractérisation et la classification des tourbes ou des composts et résidus de récolte plus ou moins transformés, (v) l'étude des relations entre matières organiques et propriétés physiques des sols. Le fractionnement granulométrique permet la séparation mécanique des fractions en vue de leur étude ultérieure (morphologique, physique, chimique et biologique). L'intérêt du fractionnement réside dans sa simplicité et sa facilité de mise en œuvre, dans son action peu dénaturante et dans son efficacité car il permet d'obtenir des fractions organiques très

\section{MATERIEL ET METHODES}

Zone d'étude : Le périmètre irrigué de Bagré se situe au Centre Est du Burkina Faso, sur le Plateau Central $\left(11^{\circ} 30^{\prime} \mathrm{N}\right.$,

$\left.0^{\circ} 25^{\prime} \mathrm{O}\right)$. Le climat est caractéristique de la zone agroécologique de savane soudano-sahélienne (BEGE, 2008). Les sols du périmètre irrigué de Bagré sont issus de formations alluviales du Quaternaire. Selon la classification de l'organisation des Nations Unies pour l'alimentation et l'agriculture (FAO, 1988), ces sols (600 ha en rive gauche) sont des Gleysols district Fluvisols (62\% de la superficie totale). La profondeur moyenne des sols se situe entre 0,4 et 1,2 m (BUNASOLS, 1994).

\section{Matériel d'étude}

Échantillons de sols: L'étude a été faite à partir d'échantillons de sols provenant d'un essai de longue différentes quant à leur morphologie, leur composition chimique et leur dynamique (Sedogo, 1993). Les méthodes de fractionnement utilisées varient en fonction de la nature du sol et de l'objectif poursuivi. La méthode de fractionnement physique de la matière organique mise au point par Feller (1979) semble plus adaptée aux conditions locales et moyens de travail et surtout aux sols sableux de la zone soudano-sahélienne (Sedogo, 1993). Son intérêt spécifique vient du fait que l'agent extractant est l'eau, ce qui permet d'éviter l'altération des différents composés organiques par les produits chimiques classiquement utilisés. La méthode permet de suivre l'évolution qualitative de la matière organique en considérant la répartition du carbone organique total et de l'azote total dans deux fractions granulométriques, les fractions dites "grossières" $(\mathrm{F}>50 \mu \mathrm{m})$ et les fractions dites "fines" ( $\mathrm{F}<50 \mu \mathrm{m})$. Les fractions grossières sont formées de matières organiques reconnaissables dites figurées (résidus d'origine végétale ou provenant d'amendements organiques non décomposés ou en cours de décomposition, parfois partiellement humifiés, débris racinaires...) alors que les fractions fines contiennent une matière organique non reconnaissable liée aux limons et argiles qui inclut la matière organique humifiée ou en voie d'humification et une partie de la biomasse microbienne. Le fractionnement granulométrique du sol permet donc l'accès à ces fractions.

durée sur l'évolution de la "matière organique" en conditions de submersion et comportant des traitements avec des apports différents de fumures organiques, minérales et organo-minérales (tableau 1). La fertilisation minérale vulgarisée "fmv" correspondait à $300 \mathrm{~kg} \mathrm{ha}^{-1} \mathrm{de}$ NPK (12:11:10) apportés au repiquage et $100 \mathrm{~kg} \mathrm{ha}^{-1}$ d'urée (saison humide) ou $150 \mathrm{~kg} \mathrm{ha}^{-1}$ d'urée (saison sèche) appliqués en deux fractions: $35 \%$ à 21 jours après repiquage (JAR) et $65 \%$ à l'initiation paniculaire. La matière organique a été apportée au labour sous forme de fumier de ferme, à la dose de $6 \mathrm{t} \mathrm{ha}^{-1} \mathrm{an}^{-1}$ (fumure organique vulgarisée ou "fov") et 12 t ha-1 an-1 (amendement organique ou "AO"). 


\section{Segda et al. J. Appl. Biosci. 2014. Le carbone et l'azote dans les différentes fractions granulométriques d'un sol brun eutrophie tropical sous irrigation de Bagré, Burkina Faso}

Tableau 1. Traitements appliqués dans les parcelles

\begin{tabular}{llc}
\hline № & Désignation & Etiquette \\
\hline Parcelles modes de gestion de la fertilisation & \\
\hline T1 & Sol sans culture et sans fertilisation & "témoin-riz" \\
T2 & Sol cultivé sans fertilisation & "témoin+riz" \\
T3 & Sol cultivé avec fumure minérale vulgarisée & "fmv" \\
T4 & Sol cultivé avec fumure organique vulgarisée & "fov" \\
T5 & Sol cultivé avec fumure organique et minérale vulgarisée & "fmv+fov" \\
T6 & Sol sans culture avec amendement organique & "AO-riz" \\
T7 & Sol cultivé avec amendement organique & "AO+riz" \\
T8 & Sol cultivé avec amendement organique et fumure minérale vulgarisée & "AO+fmv" \\
\hline
\end{tabular}

Ces échantillons ont été prélevés après quatre saisons de culture consécutives. Une parcelle adjacente au dispositif d'étude, maintenue sans culture et sans irrigation (dénommée "jachère" ou "sol de référence") a servi pour la comparaison de l'évolution qualitative de la matière organique. Le fractionnement a été réalisé en triple exemplaire pour chaque échantillon composite des huit traitements étudiés. Ces échantillons ont été prélevés à la préparation du sol en saison humide.

Méthode d'étude : La méthode de fractionnement utilisée dérive de celle de Feller (1979), adaptée par Sedogo et al. (1994) : $50 \mathrm{~g}$ de sol séché à l'air et tamisé à $2 \mathrm{~mm}$ ont été mis à agiter pendant 2 heures avec $150 \mathrm{ml}$ d'eau distillée et en présence de 5 billes de verre de $1,5 \mathrm{~cm}$ de diamètre. La suspension ainsi obtenue a été répartie, par tamisage sous l'eau, successivement en deux fractions granulométriques : une fraction dite "grossière" comprise entre 2000 et $50 \mu \mathrm{m}$ et une fraction dite "fine" inférieure à $50 \mu \mathrm{m}$. Les deux fractions ont ensuite été séchées à l'étuve à $60^{\circ} \mathrm{C}$; elles ont été récupérées par grattage et ont

\section{RÉSULTATS}

Caractéristiques physico-chimiques du sol : Le tableau 2 présente les caractéristiques physico chimiques du sol de l'expérimentation avant l'installation de l'essai. Les valeurs du pH rencontrées (moyenne 6,60) se situent dans la plage "faiblement acide à neutre". Des valeurs moyennes se rencontrent pour le carbone organique total (moyenne $0,74 \%$ pour l'horizon $0-20 \mathrm{~cm}$, et $0,49 \%$ pour l'horizon $20-50 \mathrm{~cm}$ ) et la somme des bases échangeables (SBE) dont la valeur moyenne est de 7,0 et $7,2 \mathrm{cmol} \mathrm{kg}^{-1}$ de sol respectivement pour les horizons $0-20 \mathrm{~cm}$ et 20-50 $\mathrm{cm}$. Les caractéristiques générales comme le pH sont été pesées. Les teneurs en carbone organique et en azote totaux ont été déterminées selon les méthodes d'analyses en cours au laboratoire Sols Eaux Plantes de l'INERA à Kamboinsé (Burkina Faso). Le carbone organique total a été déterminé à l'aide de la méthode Walkley and Black (1934). Le carbone est oxydé par un excès de bichromate de potassium en milieu acide sulfurique concentré. L'excès de bichromate est dosé par le sel de Mohr. L'azote total $(\mathrm{N})$ a été dosé selon la procédure décrite par Bremmer (1996). Les résultats ont été exprimés en $\mathrm{mg} / \mathrm{g}$ de fraction (teneurs) pour désigner les teneurs en carbone organique total ou azote total des fractions et en $\mathrm{mg} / \mathrm{g}$ de sol (contenus) pour désigner les quantités de carbone organique total ou d'azote total stockées dans ces fractions.

Analyse des données : Les données ont été saisies et traitées sous EXCEL 2003. L'analyse statistique descriptive de base univariée a été effectuée : calcul de la moyenne, de l'écart type et de l'étendue totale de la variable (maximum, minimum).

dans des plages favorables pour le développement du riz. De fortes valeurs de saturation du calcium échangeable $\left(\mathrm{Ca}^{2+}\right)$ et du magnésium échangeable $\left(\mathrm{Mg}^{2+}\right)$ du complexe d'échange comme les faibles valeurs du sodium $\left(\mathrm{Na}^{+}\right)$ indiquent un pouvoir tampon élevé en considérant ces deux paramètres. On constate de faibles valeurs de l'azote total ( $\mathrm{N}$ total), du potassium échangeable $\left(\mathrm{K}^{+}\right)$et du phosphore assimilable (Pass.). 
Tableau 2. Caractéristiques physico-chimiques du sol de l'expérimentation

\begin{tabular}{|c|c|c|c|}
\hline \multirow[b]{2}{*}{ Types de constituants } & \multicolumn{3}{|l|}{ Caractéristiques et composition chimique } \\
\hline & & \multicolumn{2}{|c|}{ Horizon $0-20 \mathrm{~cm}$} \\
\hline \multirow[t]{5}{*}{ Analyse granulométrique } & Argile $(<2 \mu \mathrm{m}), \%$ & 17,25 & \\
\hline & Limons fins $(2-20 \mu \mathrm{m}), \%$ & 6,75 & \\
\hline & Limons grossiers $(20-50 \mu \mathrm{m}), \%$ & 11,15 & \\
\hline & Sables fins $(50-200 \mu \mathrm{m}), \%$ & 26,53 & \\
\hline & Sables grossiers $(200-2000 \mu \mathrm{m}), \%$ & 38,33 & \\
\hline \multirow[t]{2}{*}{ Densité apparente } & d.a. $\left(\mathrm{g} \mathrm{cm}^{-3}\right)$ & 1,69 & \\
\hline & & $\begin{array}{l}\text { Horizon } \\
0-20 \mathrm{~cm}\end{array}$ & $\begin{array}{l}\text { Horizon } \\
20-50 \mathrm{~cm}\end{array}$ \\
\hline \multirow{4}{*}{ Matière organique } & Matière organique totale, \% & & \\
\hline & Carbone organique total ( $\mathrm{C}$ org.), $\%$ & 0,74 & 0,49 \\
\hline & Azote total (N tot), \% & 0,056 & 0,041 \\
\hline & $\mathrm{C} / \mathrm{N}$ & 13 & 12 \\
\hline Potassium & Total ( $\mathrm{K}$ tot), \% & 0,096 & 0,113 \\
\hline \multirow[t]{2}{*}{ Phosphore } & Assimilable ( $\mathrm{P}$ ass.), $\mathrm{mg} \mathrm{kg}^{-1}$ sol & 2,1 & 2,4 \\
\hline & Total (Ptot), $\mathrm{mg} \mathrm{kg}^{-1}$ sol & 103 & 71 \\
\hline \multirow[t]{7}{*}{ Bases échangeables } & Calcium $\left(\mathrm{Ca}^{2+}\right), \mathrm{cmol} \mathrm{kg}^{-1} \mathrm{sol}$ & 4,0 & 3,9 \\
\hline & Magnésium $\left(\mathrm{Mg}^{2+}\right), \mathrm{cmol} \mathrm{kg}^{-1} \mathrm{sol}$ & 2,8 & 3,0 \\
\hline & Potassium $\left(\mathrm{K}^{+}\right), \mathrm{cmol} \mathrm{kg}^{-1} \mathrm{sol}$ & 0,12 & 0,70 \\
\hline & Sodium $\left(\mathrm{Na}^{+}\right), \mathrm{cmol} \mathrm{kg}^{-1}$ sol & 0,16 & 0,26 \\
\hline & Somme des bases (SBE), $\mathrm{cmol} \mathrm{kg}^{-1} \mathrm{sol}$ & 7,0 & 7,2 \\
\hline & Capacité d'échange (CEC), $\mathrm{cmol} \mathrm{kg}^{-1} \mathrm{sol}$ & 7,7 & 7,9 \\
\hline & Taux de saturation, $\%$ & 91,0 & 83,1 \\
\hline \multirow[t]{2}{*}{ Réaction du sol } & pH eau & 6,1 & 6,0 \\
\hline & $\mathrm{pH} \mathrm{KCl}$ & 5,5 & 5,2 \\
\hline
\end{tabular}

Répartition granulométrique du carbone organique total et de l'azote total du sol

Carbone organique total: Les teneurs en carbone organique total ( $\mathrm{mg} \mathrm{g}^{-1}$ de fraction) des fractions sont présentées dans le tableau 3. De manière générale, les teneurs en carbone des différents traitements diminuent avec la mise en culture par rapport à la "jachère" sur l'horizon 0-20 cm, à l'exception des teneurs en carbone des traitements apportant la fumure organique vulgarisée ("fov", $6 \mathrm{t} \mathrm{ha}^{-1} \mathrm{an}^{-1}$ ) et la combinaison fumure minérale et organique vulgarisées ("fmv+fov"). Dans l'horizon 20-50 $\mathrm{cm}$ par contre, les teneurs en carbone de tous les traitements à l'exception du témoin irrigué sans riz ("témoin-riz") augmentent de 0,6 à $8,4 \%$ par rapport à la "jachère". Cependant, les variations du stock organique enregistrées avec les fumures n'affectent pas les fractions de la même manière.

Dans le sol sous "jachère" (horizon $0-20 \mathrm{~cm}$ ), la fraction grossière $(>50 \mu \mathrm{m})$ représente $7,8 \%$ du carbone organique total du sol fractionné. La teneur en carbone de la fraction baisse de manière générale au niveau de tous les traitements par rapport à la "jachère". 
Segda et al. J. Appl. Biosci. 2014. Le carbone et l'azote dans les différentes fractions granulométriques d'un sol brun eutrophie tropical sous irrigation de Bagré, Burkina Faso

Tableau 3. Effet induit des fumures sur la répartition du carbone total dans les fractions granulométriques

\begin{tabular}{|c|c|c|c|c|c|c|c|c|c|c|}
\hline & \multicolumn{2}{|c|}{$\begin{array}{l}\text { C total } \\
\text { Sol non } \\
\text { fractionné } \\
\end{array}$} & \multicolumn{2}{|c|}{$\begin{array}{l}C \text { total } \\
\text { Sol fractionné }\end{array}$} & \multicolumn{3}{|c|}{$\mathrm{F}>50 \mu \mathrm{m}$} & \multicolumn{3}{|l|}{$F<50 \mu m$} \\
\hline & $\begin{array}{l}\mathrm{mg} \mathrm{Cl} \\
\mathrm{g} \mathrm{sol}\end{array}$ & $\begin{array}{l}\% \mathrm{C} \\
\text { total }\end{array}$ & $\begin{array}{l}\mathrm{mg} \mathrm{C/} \mathrm{g} \\
\text { fraction }\end{array}$ & Indice & $\begin{array}{l}\mathrm{mg} \mathrm{Cl} \\
\text { fraction }\end{array}$ & $\begin{array}{l}\text { \% C } \\
\text { total }\end{array}$ & Indice & $\begin{array}{l}\mathrm{mg} \mathrm{C/} \mathrm{g} \\
\text { fraction }\end{array}$ & $\begin{array}{l}\% \\
\text { total }\end{array}$ & Indice \\
\hline \multicolumn{11}{|l|}{$0-20 \mathrm{~cm}$} \\
\hline "jachère" & 5,59 & 100 & 5,23 & 100,0 & 0,41 & 7,8 & 100,0 & 4,82 & 92,2 & 100,0 \\
\hline "témoin-riz" & 6,05 & 108 & 5,19 & 99,3 & 0,40 & 7,7 & 98,2 & 4,79 & 91,6 & 99,4 \\
\hline "témoin+riz" & 6,14 & 110 & 5,01 & 95,8 & 0,37 & 7,1 & 91,3 & 4,64 & 88,7 & 96,2 \\
\hline "fmv" & 6,23 & 111 & 5,02 & 95,9 & 0,30 & 5,8 & 74,7 & 4,71 & 90,1 & 97,7 \\
\hline "fov" & 6,46 & 116 & 5,44 & 103,9 & 0,39 & 7,4 & 94,8 & 5,05 & 96,5 & 104,7 \\
\hline "fmv+fov" & 6,61 & 118 & 5,24 & 100,1 & 0,39 & 7,5 & 96,2 & 4,84 & 92,6 & 100,4 \\
\hline "AO-riz" & 6,40 & 114 & 4,89 & 93,5 & 0,57 & 10,9 & 139,4 & 4,32 & 82,6 & 89,6 \\
\hline "AO+riz" & 6,15 & 110 & 5,15 & 98,5 & 0,38 & 7,3 & 93,1 & 4,77 & 91,3 & 99,0 \\
\hline "AO+fmv" & 6,26 & 112 & 5,12 & 98,0 & 0,34 & 6,5 & 82,9 & 4,79 & 91,5 & 99,3 \\
\hline \multicolumn{11}{|l|}{$20-50 \mathrm{~cm}$} \\
\hline "jachère" & 5,56 & 100 & 5,04 & 100,0 & 0,38 & 7,5 & 100,0 & 4,66 & 92,5 & 100,0 \\
\hline "témoin-riz" & 5,81 & 104 & 4,97 & 98,6 & 0,37 & 7,3 & 97,3 & 4,60 & 91,3 & 98,7 \\
\hline "témoin+riz" & 5,97 & 107 & 5,27 & 104,6 & 0,33 & 6,6 & 88,6 & 4,94 & 97,9 & 105,9 \\
\hline "fmv" & 5,93 & 107 & 5,19 & 103,0 & 0,30 & 5,9 & 79,0 & 4,89 & 97,0 & 104,9 \\
\hline "fov" & 6,18 & 111 & 5,38 & 106,8 & 0,34 & 6,7 & 89,4 & 5,05 & 100,1 & 108,2 \\
\hline "fmv+fov" & 6,32 & 114 & 5,07 & 100,6 & 0,38 & 7,6 & 101,7 & 4,68 & 92,9 & 100,5 \\
\hline "AO-riz" & 6,46 & 116 & 5,47 & 108,4 & 0,42 & 8,4 & 112,1 & 5,04 & 100,0 & 108,1 \\
\hline "AO+riz" & 6,14 & 110 & 5,29 & 104,9 & 0,39 & 7,8 & 103,6 & 4,90 & 97,2 & 105,1 \\
\hline "AO+fmv" & 6,00 & 108 & 5,12 & 101,6 & 0,39 & 7,8 & 103,8 & 4,73 & 93,8 & 101,4 \\
\hline
\end{tabular}

Fmv=Fumure Minérale Vulgarisée ; fov=Fumure Organique Vulgarisée ; $A O=A m e n d e m e n t s ~ O r g a n i q u e s$

Cependant, la teneur en carbone du traitement témoin sans riz et sans engrais ("AO-riz") représente près de $11 \%$ du carbone total du sol. Dans le sol sous "jachère" et sur l'horizon 20-50 cm, la fraction $\mathrm{F}>50 \mu \mathrm{m}$ représente $7,5 \%$ de la matière organique totale du sol fractionné. Par rapport au stock initial, sa teneur en carbone baisse de manière générale au niveau des traitements "témoin-riz" et "témoin+riz", "fmv" et "fov".

Par contre, la teneur en carbone augmente au niveau des traitements apportant de fortes quantités de matières organiques ("AO-riz", "AO+riz", "AO+fmv") et dans celui associant la fumure minérale et celle organique aux doses vulgarisées ("fmv+fov"). L'augmentation (par rapport au carbone total de la "jachère") est de $+2 \%,+12 \%,+4 \%$, et $+4 \%$ respectivement pour les traitements "fmv+fov", "AOriz", "AO+riz", et "AO+fmv".

La fraction fine $(<50 \mu \mathrm{m})$ représente plus de $92,2 \%$ de la matière organique totale du sol fractionné au niveau de la "jachère" et sur l'horizon 0-20 cm. Par rapport au stock initial, sa teneur en carbone baisse de manière générale au niveau de tous les traitements à l'exception des teneurs en carbone des traitements apportant la fumure organique vulgarisée ("fov", $6 \mathrm{t} \mathrm{ha}^{-1} \mathrm{an}^{-1}$ ) et la combinaison fumure minérale et organique vulgarisées ("fmv+fov")

Les baisses des teneurs en carbone (par rapport à la "jachère") sont de $-8,4 \%,-11,3 \%,-9,9 \%,-17,4 \%,-8,7 \%$ et $-8,5 \%$ respectivement pour les traitements "témoin-riz", "témoin+riz", "fmv", "AO-riz", "AO+riz", et "AO+fmv". Par contre, au niveau des traitements "fov" et "fmv+fov", une augmentation du carbone de $5 \%$ et $0,5 \%$ respectivement est observée. Par contre dans le sol sous "jachère" et sur l'horizon $20-50 \mathrm{~cm}$, la fraction $\mathrm{F}<50 \mu \mathrm{m}$ représente $92,5 \%$ de la matière organique totale du sol fractionné. La teneur en carbone de la fraction baisse seulement au niveau du traitement "témoin-riz" (baisse de 1,3\%). Par contre, une augmentation au niveau de tous les autres traitements par rapport au stock est observée, augmentation qui va de $+0,5$ à $+8,2 \%$. La presque totalité de la matière organique se trouve accumulée dans I'horizon inférieur $(20-50 \mathrm{~cm})$ et dans la fraction organominérale. Ce résultat indique une augmentation des teneurs en carbone par rapport à la "jachère", et permet d'émettre l'hypothèse d'une accumulation de matière 
organique après la mise en culture dans les conditions de riziculture irriguée.

Azote total: Les teneurs en azote total $\left(\mathrm{mg} \mathrm{g}^{-1}\right.$ de fraction) des fractions sont présentées dans le tableau 4. De manière générale, les teneurs en azote total des différents traitements diminuent avec la mise en culture par rapport à la jachère sur l'horizon $0-20 \mathrm{~cm}$, à l'exception des teneurs en azote des traitements apportant la fumure minérale vulgarisée ("fmv"). Dans l'horizon 20-50 cm par contre, les teneurs en azote total de tous les traitements (à l'exception du "témoin-riz") augmentent de 3,4 à 16,5\% par rapport à la "jachère" Mais comme pour le carbone organique total, les variations du stock en azote enregistrées avec les fumures n'affectent pas les fractions de la même manière.

Tableau 4. Effet induit des fumures sur la répartition de l'azote total dans les fractions granulométriques

\begin{tabular}{|c|c|c|c|c|c|c|c|c|}
\hline & \multicolumn{2}{|c|}{$\begin{array}{l}\mathrm{N} \text { total } \\
\text { Sol fractionné }\end{array}$} & \multicolumn{3}{|l|}{$\mathrm{F}>50 \mu \mathrm{m}$} & \multicolumn{3}{|l|}{$\mathrm{F}<50 \mu \mathrm{m}$} \\
\hline & $\begin{array}{l}\mathrm{mg} \mathrm{N} / \\
\text { fraction }\end{array}$ & g Indice & $\begin{array}{l}\mathrm{mg} \mathrm{N} / \\
\text { fraction }\end{array}$ & $\begin{array}{c}g \% \mathrm{~N} \\
\text { total }\end{array}$ & Indice & $\begin{array}{l}\mathrm{mg} \mathrm{N} / \mathrm{g} \\
\text { fraction }\end{array}$ & $\begin{array}{l}\% \\
\text { total }\end{array}$ & $\mathrm{N}$ Indice \\
\hline \multicolumn{9}{|l|}{$0-20 \mathrm{~cm}$} \\
\hline "jachère" & 0,46 & 100,0 & 0,06 & 12,5 & 100,0 & 0,40 & 87,6 & 100,0 \\
\hline "témoin-riz" & 0,35 & 75,9 & 0,09 & 20,4 & 163,0 & 0,26 & 55,6 & 63,4 \\
\hline "témoin+riz " & 0,38 & 83,3 & 0,07 & 14,7 & 117,8 & 0,32 & 68,6 & 78,3 \\
\hline "fmv" & 0,51 & 110,3 & 0,09 & 19,7 & 157,7 & 0,42 & 90,6 & 103,4 \\
\hline "fov" & 0,42 & 92,3 & 0,13 & 29,2 & 233,6 & 0,29 & 63,1 & 72,0 \\
\hline "fmv+fov" & 0,37 & 80,8 & 0,12 & 26,2 & 209,4 & 0,25 & 54,6 & 62,3 \\
\hline "AO-riz" & 0,42 & 91,8 & 0,09 & 19,7 & 157,7 & 0,33 & 72,1 & 82,3 \\
\hline "AO+riz" & 0,35 & 76,4 & 0,08 & 18,5 & 147,8 & 0,27 & 57,9 & 66,1 \\
\hline "AO+fmv" & 0,34 & 74,6 & 0,07 & 15,5 & 124,3 & 0,27 & 59,1 & 67,5 \\
\hline \multicolumn{9}{|l|}{$20-50 \mathrm{~cm}$} \\
\hline "jachère" & 0,60 & 100,0 & 0,10 & 16,8 & 100,0 & 0,50 & 83,3 & 100,0 \\
\hline "témoin-riz" & 0,55 & 91,2 & 0,11 & 18,3 & 108,9 & 0,44 & 72,9 & 87,5 \\
\hline "témoin+riz" & 0,67 & 111,5 & 0,11 & 17,7 & 105,6 & 0,56 & 93,8 & 112,5 \\
\hline "fmv" & 0,68 & 112,9 & 0,11 & 17,8 & 106,1 & 0,57 & 95,1 & 114,1 \\
\hline "fov" & 0,62 & 103,4 & 0,10 & 16,9 & 100,8 & 0,52 & 86,5 & 103,8 \\
\hline "fmv+fov" & 0,66 & 110,7 & 0,10 & 16,9 & 100,8 & 0,56 & 93,8 & 112,5 \\
\hline "AO-riz" & 0,70 & 116,5 & 0,12 & 20,7 & 123,0 & 0,58 & 95,8 & 115,0 \\
\hline "AO+riz" & 0,68 & 113,2 & 0,11 & 18,1 & 107,8 & 0,57 & 95,1 & 114,1 \\
\hline "AO+fmv" & 0,70 & 116,2 & 0,10 & 17,2 & 102,6 & 0,59 & 99,0 & 118,8 \\
\hline
\end{tabular}

La fraction fine $(<50 \mu \mathrm{m})$ représente $87,5 \%$ de l'azote total du sol fractionné dans le sol sous "jachère" et sur l'horizon 0-20 cm. Par rapport au stock initial, la teneur en azote total de la fraction augmente seulement au niveau du traitement "fmv" (+3,4\%). Par contre, une diminution des teneurs en azote total par rapport à la jachère est observée pour tous les autres traitements. Les diminutions en azote total par rapport à la "jachère" sont de $-36 \%$, $22 \%,-28 \%,-38 \%,-18 \%,-34 \%$, et $-33 \%$ respectivement au niveau du "témoin-riz", "témoin+riz", "fov", "fmv+fov", "AOriz", "AO+riz", et "AO+fmv".
Dans le sol sous "jachère" et sur l'horizon $20-50 \mathrm{~cm}$, la fraction $\mathrm{F}<50 \mu$ m représente $83,2 \%$ de l'azote total du sol fractionné. Contrairement à l'horizon supérieur, la teneur en azote total de la fraction augmente de manière générale au niveau de tous les traitements à l'exception du témoin-riz, augmentations qui se situent entre $4 \%$ et $19 \%$. Les surplus en azote total par rapport à la "jachère" sont de $+13 \%,+14 \%,+4 \%,+13 \%,+15 \%,+14 \%$, et $+19 \%$ et respectivement au niveau du "témoin+riz", "fmv", "fov", "fmv+fov", "AO-riz", "AO+riz", et "AO+fmv". 


\section{DISCUSSION}

Cette étude sur le fractionnement physique de la matière organique a montré que deux ans après leur mise en culture, il y a une accumulation du carbone et de l'azote dans les sols de rizière de Bagré, par rapport à la jachère. Ces résultats ne sont pas en conformité avec ceux de Bacyé (1993), Sedogo (1993), Kambire et al. (2001), Bilgo (2005) dont les travaux ont concerné des échantillons de sols prélevés dans des parcelles de cultures pluviales. La baisse du taux de matière organique des sols tropicaux exondés qui survient dès leur mise en culture ainsi que la dégradation rapide de certaines propriétés du sol (structure, activité biologique...) consécutive à la mise en culture sont attribuées à l'évolution des fractions grossières (par exemple Sedogo et al. (1994), Kambiré et al. (2001). En effet, ces auteurs ont montré que c'est la fraction grossière qui constitue le compartiment le plus labile, du fait que le rapport $\mathrm{C} / \mathrm{N}$ de la matière organique de ces fractions est relativement plus élevé (Feller et al. 1991b) que celui de la fraction organo minérale. II est généralement admis en effet que ce sont les fractions jeunes et labiles de la matière organique du sol qui contribuent plus aux processus de minéralisation et d'immobilisation que les fractions de matière organique vieille et plus stable (Sedogo et al. 1994; Kambire et al. 2001; Bilgo, 2005). La fraction grossière constitue par conséquent une source de carbone et d'énergie pour les microorganismes. Nos résultats montrent cependant que c'est la fraction organo-minérale qui semble constituer plutôt une source de carbone et d'énergie pour les microorganismes du sol, le rapport $\mathrm{C} / \mathrm{N}$ de cette fraction étant plus élevé $(\mathrm{C} / \mathrm{N}=11$ à 18$)$ que celui de la fraction grossière $(\mathrm{C} / \mathrm{N}=3$ à 4$)$. Dans les sols submergés (comme c'est le cas à Bagré), plusieurs travaux de recherche ont permis d'expliquer l'accumulation de la matière organique. Pour Esteves et al. (2001), la décomposition de la matière

\section{CONCLUSION}

La mise en culture sous irrigation conduit à une accumulation de la matière organique dans les sols de Bagré. La plupart des études sur la matière organique dans le monde ont été effectuées principalement sur l'horizon superficiel $(0-10 \mathrm{~cm}, 0-15 \mathrm{~cm}$, ou 0-20 cm). Cependant, nos résultats indiquent que l'accumulation de la matière organique est plus importante dans les horizons inférieurs $(20-50 \mathrm{~cm})$. Dans les sols inondés, compte tenu des différents mécanismes qui interviennent dans l'accumulation de la matière organique et de la lixiviation organique à travers les processus de respiration et de fermentation en anaérobiose est métaboliquement moins efficiente et conduit à des décompositions lentes des substrats organiques et partant, à une accumulation nette de ces composés organiques. Selon Mathieu et al. (2002), la culture intensive dans les rizières submergées entraîne une diminution de la quantité de fer libre dans le sol. II en résulte que les sols sous submersion continue et cultivés intensivement deviennent déficients en fer ferrique ou accepteur d'électrons, conduisant à la baisse des taux d'oxydation et de minéralisation de l'azote organique (Sahrawat, 2002). Le niveau de l'horizon peut aussi expliquer la variabilité de la concentration du carbone du sol (Powers \& Schlesinger, 2002), car les argiles des horizons inférieurs emprisonnent fortement le carbone qui ne peut être oxydé par la méthode Walkley et Black. Dans la Vallée du Fleuve Sénégal, Haefele et al. (2004) ont observé une augmentation des teneurs en carbone et en azote dans les sols de rizière, la matière organique pénétrant de $0,5 \mathrm{~m}$ (site de Fanaye, $16^{\circ} 33^{\prime} \mathrm{N}-15^{\circ} 46^{\circ} \mathrm{O}$ ) à $0,8 \mathrm{~m}$ de profondeur (site de Ndiaye, $16^{\circ} 14^{\prime} \mathrm{N}-16^{\circ} 14^{\prime} \mathrm{O}$ ). Sur le site de Fanaye, la pénétration de la matière organique à $0,5 \mathrm{~m}$ de profondeur correspondrait à la profondeur des fentes de retrait apparues pendant l'inter campagne (la période de jachère sèche entre deux campagnes rizicoles). Par contre à Ndiaye, ces auteurs expliquent que la pénétration serait liée à l'infiltration de la matière organique dissoute avec l'eau de percolation. En Chine, Zhang \& He (2004) ont montré qu'avec le temps, l'accumulation du carbone et de l'azote s'effectuait sur le profil jusqu'à $1,5 \mathrm{~m}$ de profondeur. Pour ces auteurs, les quantités importantes de carbone, d'azote et de phosphores accumulés dans les sols de rizière constituent un potentiel dans la conservation de ces éléments.

de la matière organique dissoute, les études devraient concerner tout le profil cultural. II est important de comprendre et de déterminer les facteurs responsables de l'accumulation de la matière organique et les mécanismes qui y contribuent. Les sols de rizières de Bagré offrent un excellent exemple de conservation, de maintien et de stockage de la matière organique. De ce fait, la riziculture irriguée pourrait contribuer dans la séquestration $d u$ carbone atmosphérique. 

granulométriques d'un sol brun eutrophie tropical sous irrigation de Bagré, Burkina Faso

\section{BIBLIOGRAPHIE}

Bacyé B., 1993. Influence des systèmes de culture sur l'évolution du statut organique et minéral des sols ferrugineux et hydromorphes de la zone soudano-sahélienne (Province du Yatenga, Burkina Faso). Doct. Thesis University Aix Marseille III, France, $243 p$.

BEGE, 2008. Étude de faisabilité intégrée sur la biomasse des vallées du Sourou et de Bagré pour la production d'éthanol et d'électricité. Rapport final de consultation. Ministère des Mines et de l'Energie, Ouagadougou, $183 \mathrm{p}$.

Bhattacharyya R., Prakash V., Kundu S., Srivastva A.K., Gupta H.S., Mitra S., 2009. Long term effects of fertilization on carbon and nitrogen sequestration and aggregate associated carbon and nitrogen in the Indian sub-Himalayas. Nutrient Cycling in Agroecosystems 86 (1):1-16.Bilgo A., 2005. Statut organo-minéral et biologique des sols dans les systèmes culture-jachère naturelle de courte durée ou améliorée à Andropogon gayanus Kunth. Cas de Bondoukuy en zone sud soudanienne du Burkina Faso. Thèse de Doctorat Unique en Sciences Biologiques Appliquées, Option Biologie et Écologie Végétales, Université de Ouagadougou, $177 \mathrm{p}$.

Bremmer J.M., 1996. Nitrogen-Total. In: Methods of soil analysis. Part 3, Chemical methods. Bigham, J.M (Ed.), Soil Science Society of America/American Society of Agronomy, Madison, USA, p. 10851122.

BUNASOLS., 1990. Manuel pour l'évaluation des terres. Document Technique $n^{\circ}$ 6, $181 \mathrm{p}$.

BUNASOLS., 1994. Étude pédologique du périmètre irrigué de Bagré (Bagré aval, bief $A$ et $B$, première tranche), échelle 1/10 000 è. Volume 1 : rapport principal, Rapport technique 95, BUNASOLS, Ouagadougou, $75 \mathrm{p}$.

Christensen B.T., 1992. Physical Fractionation of Soil and Organic Matter in Primary Particle Size and Density Separates, Advances in Soil Science, Vol. 20, pp. 1-90.

Creamer C.A., Filley T.R., Boutton T.W., 2013. Long-term incubations of size and density separated soil fractions to inform soil organic carbon decay dynamics. Soil Biol. Biochem. 57: 496-503

Elliott E.T. et Cambardella C.A., 1991 - Physical separation of soil organic matter. Agriculture, Ecosystems and Environnement, 34, 407-419.

Esteves F.A., Enrich-Prast A., Biesboer D.D., 2001. Potential denitrification in submerged natural and impacted sediments of Lake Batata, an Amazonian lake. Hydrobiologica 444: 111-117.

FAO (Food and Agriculture Organisation of the United Nations), 2002. La séquestration du carbone pour une meilleure gestion des terres. Rapport sur les ressources en sol du monde 96, FAO, Rome

FAO (Food and Agriculture Organisation of the United Nations), 1988. FAO-UNSECO Soil Map of the World. Revised legend, World Soil Resources Report 60, FAO, Rome.

Feller C., 1979. Une méthode de fractionnement granulométrique de la matière organique des sols. Application aux sols tropicaux à textures grossières, très pauvres en humus. Cah. ORSTOM. sér. Pédol., Vol XVII (4): 339-346.

Feller C. and Beare M.H., 1997. Physical control of soil organic matter dynamics in the tropics. Geoderma 79: 69-116.

Feller C., François C., Villemin G., Portal J.M., Toutain F. et Morel J.L., 1991a. Nature des matières associées aux tractions argileuses d'un sol ferrallitique. C.R. Acad. Sci., Paris, 312, sér. 11: 1491-1497.

Feller C., Fritsch E., Poss R. Valentin C., 1991b. Effet de la texture sur le stockage et la dynamique des matières organiques dans quelques sols ferrugineux et ferrallitiques (Afrique de l'Ouest, en particulier). Cah.ORSTOM, sér.Pédol. Vol XXVI (1) : 25-36.

Ge S., Xu H., Ji M., Jiang Y., 2013. Characteristics of Soil Organic Carbon, Total Nitrogen, and C/N Ratio in Chinese Apple Orchards. Open Journal of Soil Science, 2013 (3): 213-217

Haefele S.M., Wopereis M.C.S., Schoenbohm A., Wiechmann H., 2004. Long-Term Fertility Experiments for Irrigated Rice in the West African Sahel: Effect on soil characteristics. Field Crops Research 85 (1): 61-77.

IPCC., 2008. Climatic Change 2007: The Physical Science Basis-Summary for Policymakers. Contribution of Working Group I to the fourth Assessment Report of the International Panel on Climate Change (eds S. Solomon et al.). Cambridge University Press, Cambridge.

Jenkinson DS, Adams DE, Wild A (1991). Model estimates of $\mathrm{CO}_{2}$ emissions from soil in response to global warming. Nature 351:304-306.

Kambiré S.H., Sédogo M.P. Hien V., 2001. Effet de l'âge de mise en culture sur la dynamique des compartiments granulométriques de la matière 


\section{Segda et al. J. Appl. Biosci. 2014. Le carbone et l'azote dans les différentes fractions}

granulométriques d'un sol brun eutrophie tropical sous irrigation de Bagré, Burkina Faso

organique d'un sol ferrugineux tropical du Burkina Faso. Sci. et Tech. Sér. Sci. Nat. 25 (1): 7-15.

Li P., T. L. Zhang, C. Y. Jiang and Y. P. Che., 2011. Discrepancy in Response of Rice Yield and Soil Fertility to Long-Term Chemical Fertilization and Organic Amend- ments in Paddy Soils Cultivated from Infertile Upland in Subtropical China. Agricultural Sciences in China, Vol. 10, No. 2, pp. 259-266.

Mahieu N., Olk D.C., Randall E.W., 2002. Multinuclear magnetic resonance analysis of two humic acid fractions from lowland rice soils. J. Environ. Qual. 31: 421-430.

Nacro H.B., Benest D. Abbadie L., 1996. Distribution of microbial activities and organic matter according to particle size in a humid savanna soil (Lamto, Côte d'Ivoire). Soil Biol. Biochem. 28 (12): 16871697.

Powers J.S. and Schlesinger W.H., 2002. Relationships among soil carbon distributions and biophysical factors at nested spatial scales in rain forests of north-eastern Costa Rica. Geoderma 109 (3-4): 165- 190.

Sahrawat K.L. 2002. Reducible iron affects organic matter oxidation and ammonium production in submerged soils and sediments. Current Science 83: 1434-1435

Sédogo P.M., 1993. Evolution des sols ferrugineux tropicaux lessivés sous culture: incidence des modes de gestion sur la fertilité. Thèse doctorat es sciences, Université Nationale de Côte d'Ivoire. 333 pp.

Sédogo P.M., Lompo F., Ouattara B., 1994. Le carbone et l'azote dans les différentes fractions granulométriques d'un sol ferrugineux tropical : effets de quatre types d'amendements organiques. Sci. et Tech. Sér. Sci. Nat. 21 (1): 114-124.

Segda Z., Bonzi M., Gnankambary Z., Lompo F., Sedogo M.P., 2014. Influence of soil fertility management on organic carbon mineralization in irrigated rice. J. Agric. Crop Res 2 (2) : 32-43

Segda Z., 2006. Gestion intégrée de la fertilité du sol pour une production améliorée et durable du riz irrigué (Oryza sativa L.) au Burkina Faso. Cas de la plaine irriguée de Bagré. Thèse de Doctorat Unique en Sciences Biologiques Appliquées, Option Biologie - Écologie Végétales, Université de Ouagadougou, 164 pp.

Sombrero A., Benito de A., 2010. Carbon accumulation in soil : ten year study of conservation tillage and crop rotation in a semi-arid area of Castile-Leon, Spain. Soil and Tillage Research $107:$ 64-70

Sui Y.Y., X. Y. Zhang, X. G. Jiao, Q. C. Wang and Zhao J., 2005. Effect of Long-term Different Fertilizer Applications on Organic Matter and Nitrogen of Black Farmland. Journal of Soil and Water Conservation, Vol. 19 (6): 190-192.

Valentini R, Matteucci G, Dolman H., 2000. Respiration as the main determinant of carbon balance of European forests. Nature 404: 861-865.

Walkley A. and Black J. A., 1934. An examination of the Degtjareff method for determining soil organic matter and proposed modification of the chromatic acid titration method. Soil Science 37: 29-38.

Wang W.J, Chalk P.M, Chen D., Smith C.J, 2001. Nitrogen mineralization, immobilization and loss, and their role in determining differences in net nitrogen production during waterlogged and aerobic incubation of soils. Soil Biol. Biochem. 33: $1305-1315$.

Zhang M. et He Z., 2004. Long-term changes in organic carbon and nutrients of an Ultisol under rice cropping in southeast China. Geoderma 118: 167-179.

Zhang C.H., Z. M. Wang, W. M. Ju., Ren C. Y., 2011. Spatial and Temporal Variability of Soil C/NRatio in Songnen Plain Maize Belt. Environmental Science, Vol. 32 (5): 1407-1414. 